\title{
¿SE PUEDE GENERAR CONOCIMIENTO MEDIANTE LA GESTIÓN ADAPTATIVA PARA FUNDAMENTAR UN CAMBIO DE PARADIGMA DE LA SELVICULTURA?
}

\author{
Felipe Bravo Oviedo ${ }^{1,2}$ \\ ${ }^{1}$ Instituto Universitario de Gestión Forestal Sostenible, Universidad de Valladolid-INIA \\ ${ }^{2}$ Escuela Técnica Superior de Ingenierías Agrarias, Universidad de Valladolid. Campus de Palencia.Avda. \\ Madrid 44. 34071-PALENCIA (España). Correo electrónico: fbravo@pvs.uva.es
}

\section{Resumen}

La selvicultura, que se desarrolló hace más de doscientos años para atender las necesidades sociales respecto de productos escasos con una demanda alta, tiene hoy el reto de adaptar las herramientas disponibles, y desarrollar otras nuevas, para cubrir la variada demanda de bienes y servicios asegurando la persistencia de las masas forestales y evitando la irreversibilidad de las actuaciones. La nueva situación que supone el cambio ambiental y social hace preciso un cambio de paradigma en la ciencia forestal en general y en la selvicultura en especial. Dado los plazos de respuesta de los sistemas forestales los métodos tradicionales de investigación deben ser complementados con estrategias de adquisición de conocimiento basadas en la gestión adaptativa. A lo largo de esta ponencia se pasará revista a los conceptos básicos de la selvicultura, los aspectos fundamentales que debe abordar la selvicultura para asentar el cambio de paradigma preciso, se exploraran los límites de la gestión tradicional y del método científico y las oportunidades de la gestión adaptativa para alcanzar este objetivo.

Palabras clave: Ciencia, Forestal, Cambio global, Gestión adaptativa

\section{INTRODUCCIÓN}

La selvicultura como ciencia y como técnica se desarrolló hace más de doscientos años para atender las necesidades sociales en un entorno de productos escasos con una demanda alta (maderas, leñas,..) Los fundamentos y procedimientos desarrollados fueron exitosos asegurando la producción sostenida de los bienes demandados y obteniendo como un preciado subproducto un conjunto de servicios ambientales. En la actualidad las sociedades, en un entorno en el que la sostenibilidad (ambiental, social y económica) es un requisito, demandan de los bosques un amplio abanico de funciones (bienes y servicios) pasando a ser, en algunos casos, los bienes tradicionales un subpro- ducto valioso. El reto de los forestales hoy es adaptar las exitosas herramientas para cubrir las demandas anteriores al nuevo escenario asegurando la persistencia de las masas forestales y evitando la irreversibilidad de las actuaciones.

Dada la trayectoria histórica de la profesión forestal no es extraño que PuETTMAnN et al. (2009) establezcan que para la mayoría de los forestales la sostenibilidad sea equivalente a rendimiento sostenido y que indiquen que domina la idea de que lo que es bueno para los árboles es bueno para los bosques. Aunque esto sea matizable, por ejemplo, en el caso de la selvicultura mediterránea con una base multifuncional desde antiguo, no debe extrañar que la preponderancia del árbol como proveedor de bienes y servicios sea criticada. 
Durante las últimas décadas, se ha producido un incremento de la preocupación en todo el mundo en relación con la conservación y gestión de los recursos naturales. Esta tendencia se ha manifestado en diversas iniciativas internacionales como la Conferencia de Rio de Janeiro de 1992. Así se definió la necesidad de incorporar medidas de sostenibilidad en la gestión forestal para asegurar tanto el uso racional de los recursos naturales como la persistencia en el tiempo de los valores ambientales de los mismos. Es claro que la selvicultura aplicada en los bosques templados, y en particular en Europa, cumplían en muchos casos estas medidas pero también lo es que no era así en otros tipos de bosques. Esta iniciativa tuvo una gran repercusión tanto en la política mundial, a través de la Reunión de Johannesburgo en 2002 (conocida como RIO+10) o en Rio de Janeiro en 2012 (Rio+20), como en Europa donde se había desarrollado con anterioridad la Conferencia de Estrasburgo (1990) y posteriormente se realizaron las de Helsinki (1993), Lisboa (1998), Viena (2003), Varsovia (2007) y Oslo (2011) así como la prevista en Madrid a finales de 2015. En estos eventos, los países europeos han alcanzado sucesivos acuerdos para promover la cooperación en la protección de los bosques y en la gestión forestal sostenible (http://www.foresteurope.org). Aproximadamente en las mismas fechas se desarrollaron otras iniciativas que intentaban definir los objetivos de la sostenibilidad en los sistemas forestales así como sus niveles apropiados. Por tanto, una serie de criterios e indicadores de gestión forestal sostenible promovidos por grupos de interés como el Forest Stewardship Council (FSC) o la Red Internacional de Bosques Modelo o mediante acuerdos gubernamentales como el Proceso de Montreal (para los bosques templados y boreales) o el proceso de Tarapoto (para los bosques de la Cuenca del Amazonas)

Aunque las condiciones en que se desarrolla la selvicultura han cambiado los conceptos básicos de la selvicultura como ciencia siguen estables y son los siguientes (modificado a partir de LEARY, 1985): (1) Interacción (competencia, facilitación,...), (2) Estación (productividad, capacidad de carga,..), (3) Densidad (número de individuos, espaciamiento,...) y (4) Crecimiento y Dinámica (incremento de tamaño, mortalidad, incorporación de individuos,...) La manipula- ción práctica de estos conceptos es lo que da potencia a la selvicultura como técnica

Un aspecto crucial para desarrollar nuevos métodos de gestión forestal sostenible y para controlar su ejecución es implementar sistemas de gestión adaptativa que permitan adaptar la gestión forestal al cambio global en sus vertientes ambiental, social y económica. El objetivo de este trabajo es plantear elementos para discutir el papel de la ciencia en el desarrollo de nuevo conocimiento selvícola. El reto es adaptar las herramientas disponibles y desarrollar nuevas asegurando la persistencia de las masas forestales y evitando la irreversibilidad de los efectos de las actuaciones.

El viejo adagio forestal Saber es hacer, acuñado por Bernado de la Torre en 1846, dio paso al lema del Congreso Forestal Español de 2009: Saber qué hacer. Sin embargo el hacer sin un marco conceptual sirve de poco tal y como lo expresó DEMING (1993), ingeniero y estadístico estadounidense, "Experience by itself teaches nothing... Without theory, experience has no meaning. Without theory, one has no questions to ask. Hence, without theory, there is no learning. [La experiencia por sí misma no enseña nada.... Sin teoría, la experiencia no tiene significado. Sin teoría, uno no tiene preguntas que hacer. Por lo tanto, sin teoría no hay aprendizaje]".

\section{PREGUNTAS CLAVES PARA LA GESTIÓN FORESTAL QUE DEBE RESPONDER LA CIENCIA FORESTAL}

Desde mi punto de vista las preguntas relevantes que debe responder la Ciencia Forestal en un máximo de 10 años son las siguientes:

1. ¿Cuáles son las estrategias selvícolas más adecuadas para asegurar la persistencia y funcionamiento de los sistemas forestales en bajo condiciones cambiantes tanto a nivel ambiental como socioeconómico?

2. ¿Cuál es la respuesta más adecuada frente a la amenaza que suponen las especies invasoras o potencialmente invasivas o los brotes de nuevas plagas o enfermedades forestales?

3. ¿De qué forma los productos y servicios (tradicionales o novedosos) que generan los bosques y que son valorados (aunque muchas veces no pagados) por la sociedad 
pueden ayudar a mantener el nivel adecuado de selvicultura?

4. ¿Cómo pueden los bosques y la gestión forestal colaborar (mediante la provisión de bienes y servicios) en el desarrollo de modelos económicos basados en la sostenibilidad (Green Economy)? (biomasa, maderas de calidad, mantenimiento de la biodiversidad, los árboles como biorefinerías, intervenciones autofinanciadas, ecoturismo,...)

5. ¿Cuáles son los instrumentos de bajo costo más adecuados para la implementación de métodos de gestión y planificación forestal dentro de un marco de desarrollo económico basado en el conocimiento (Knowledge Based BioEconomy)? (LiDAR, Software libre, entornos 2.0, toma de decisiones multicriterio, participación social,...)

Estas preguntas tiene un sesgo claramente personal pero el objeto de esta ponencia no es discutir qué preguntas son relevantes si no presentar ideas para que el avance científico sea rápido de forma que un nuevo paradigma de ciencia forestal pueda asentarse firmemente y permita sustentar una gestión forestal acorde con las nuevas demandas sociales. En cualquier caso la investigación selvícola debería centrarse en las teorías generales e investigar la respuesta básica para desarrollar generalizaciones conceptuales basadas en la comprensión de los procesos subyacentes a las respuestas a los tratamientos (PuetTMANN et al., 2009). Hacemos demasiados estudios de casos y poca teoría científica lo que limita el impacto y la aplicación de los resultados científicos.

\section{LOS LÍMITES DE LA GESTIÓN TRADI- CIONAL Y DEL MÉTODO CIENTÍFICO PARA EL CAMBIO DE PARADIGMA DE LA CIENCIA FORESTAL}

Muchos de los problemas que a continuación se describen no son achacables solo a la gestión tradicional o a la aplicación del método científico en la ciencia forestal. Como se verá en muchos casos son compartidos. Por tanto, la solución a estos límites solo podrá ser compartidas ya que la ciencia forestal es necesariamente aplicada.

\section{Límites de la gestión forestal tradicional}

En muchos casos los límites económicos hacen que sea necesario simplificar la aplicación de la selvicultura de forma que se intente basar ésta en tipos de bosques y esquemas de tratamientos selvícolas denominados modelos selvícolas. Mediante el uso de tipologías y de los modelos selvícolas y sus protocolos de implementación se pretende aplicar una aproximación reduccionista a sistemas complejos como los forestales. Esta aproximación no tiene en cuenta diversos aspectos claves de los sistemas forestales como son las propiedades emergentes, la heterogeneidad espacial y temporal o la escala de trabajo (dando una preponderancia alta al nivel de rodal) lo que hace que en algunos casos los resultados de las actividades selvícolas no sean los esperados.

Además la formación forestal, centrada en la resolución de problemas ya conocidos y no en el planteamiento de problemas con solución incierta, hace que, a menudo, la investigación se vea como un complemento de la profesión y no como el núcleo de las disciplinas forestales.

La definición que se hace de la selvicultura no es ajena a los problemas en el avance de la ciencia forestal. La selvicultura se define de acuerdo con Helms (1998) como "El arte y la ciencia de controlar el establecimiento, crecimiento, composición, sanidad y calidad de los bosques y las masas forestales para cumplir diversas necesidades y valores de los propietarios y la sociedad de una forma sostenible". Otra definición fue planteada por BRAVo (2007) "La selvicultura es la ciencia y la técnica relacionada con la intervención razonada del hombre en el bosque, mediante el control de su establecimiento, crecimiento y composición, y cuya finalidad es satisfacer las diversas necesidades y aspiraciones de los propietarios y la sociedad garantizando en todo momento la persistencia de los ecosistemas forestales". Finalmente el Diccionario Español de Ingeniería (REAL ACADEMIA DE INGENIERÍA, 2014) define la selvicultura como "Teoría y práctica sobre el establecimiento, desarrollo, composición, sanidad, calidad, aprovechamiento y regeneración de las masas forestales, para satisfacer las diversas necesidades de la sociedad, de forma continua o sostenible". De acuerdo con PuettMann et al. (2009) al definir la selvicultura como arte se 
puede pensar que la experiencia obtenida por años de práctica así como la observación cuidadosa puede llevar al conocimiento preciso para modificar medidas selvícolas del pasado para responder adecuadamente a nuevas condiciones de forma que el llamado "ojo del forestal" deviene en instrumento clave para diagnosticar y modificar los sistemas selvícolas. La definición de la selvicultura como arte, y su corolario del "ojo del forestal" es uno de los mayores obstáculos al desarrollo de la ciencia forestal

El peso de la tradición y del concepto del "ojo del forestal" lleva a la aplicación, quizás rutinaria, de prácticas adaptadas a las condiciones locales pero no promueve en la educación forestal la introducción de ideas rutinarias (PUETTMANN et al., 2009) alejando el pensamiento crítico de la selvicultura. Así PuetTMANN et al. (2009) indican que el enfásis en la tradición forestal es probablemente una de las razones por las que la selvicultura no se adapta fácilmente a los cambios sociales, ambientales o económicos. Además de esto habría que añadir el largo plazo de respuesta de los sistemas forestales a los tratamientos selvícolas debido a la longevidad de los árboles. En cualquier caso, se debe resaltar que la tradición forestal es un valor de la profesión pero no debe impedir su desarrollo en un entorno cambiante.

\section{Límites del sistema científico}

La capacidad de investigación, de un sector o país, es la magnitud de la habilidad para desarrollar, generar y diseminar ciencia y tecnología (NATIONAL RESEARCH COUNCIL, 2002) La separación entre la gestión y la investigación forestal, y la fragmentación y aislamiento de los grupos de investigación, disminuye nuestra capacidad de investigación y hace que los esfuerzos realizados no cristalicen en un verdadero avance mediante la aplicación práctica de los hallazgos y desarrollos científicos y tecnológicos. El seguimiento, la investigación y la aplicación de nuevas tecnologías debe ser la base para conservar, mejorar y restaurar los sistemas forestales (NATIONAL RESEARCH COUNCIL, 2002)

Uno de los aspectos clave es la extensibilidad de los resultados de investigación de forma que los hallazgos obtenidos puedan ser de aplicación más general. Sin embargo, la mayoría de los experimentos forestales se hacen en parcelas relativamente pequeñas y homogéneas internamente con un número de replicas limitado y con un seguimiento temporal no muy extenso. Las parcelas pequeñas tienen indudables ventajas (Puettmann et al., 2009) ya que (1) es más fácil encontrar condiciones homogéneas, (2) se pueden hacer más unidades experimentales y (3) se optimiza la inversión (dinero, trabajo,...) en los experimentos. Sin embargo estas ventajas conllevan problemas al trasladar los resultados a escala operativa (PUETTMAnN et al., 2009) como, por ejemplo, no tener en cuenta la heterogeneidad de las masas forestales o que los tratamientos selvícolas se aplican de forma menos cuidadosa en los trabajos rutinarios que en los expermientales.

En el caso de los sistemas forestales los problemas de escala (con una atención desmedida tanto en la investigación como en la gestión en la escala del rodal) como de los límites (poca atención a los ecotonos) y de los plazos (largo tiempo de desarrollo de los bosques debido a la longevidad y tasa de crecimiento de los árboles que hacen que los cambios de prioridades de gestión y de demandas sociales no puedan ser atendidos de forma ágil) no hacen sino añadir complejidad a los problemas que se pretenden abordar.

\section{GESTIÓN FORESTAL ADAPTATIVA COMO HERRAMIENTA DE INVESTIGACIÓN}

\section{¿Qué es la gestión forestal adaptativa?}

La idea de gestión adaptativa como una estrategia útil para la gestión de los recursos naturales se debe a Holling (1978). De acuerdo con la definición propuesta por NYBERG (1998), la gestión adaptativa es un proceso sistemático para la continua mejora de las prácticas de gestión mediante el aprendizaje a partir de los resultados de la actividad selvícola. Aunque se podría pensar que la implementación de planes de gestión (es sus diferentes formatos y denominaciones: proyectos de ordenación, planes técnicos, planes dasocráticos,...) y su seguimiento cuidadoso sería equivalente a la gestión adaptativa. Nada más lejos de la realidad. Las principales características de la gestión adaptativa son las siguientes (basado en NYBERG, 1998; STANKEY et al., 2005): 
1. Reconocimiento de la incertidumbre sobre cuál es el método de gestión más adecuado para un situación o contexto dado.

2. Se realizan esfuerzos destinados a integrar los conocimientos de diferentes disciplinas en los métodos de gestión y en modelos dinámicos de forma que se pueda predecir el impacto de diferentes alternativas.

3. Selección cuidadosa de las prácticas y métodos a ensayar, eliminando las opciones que tienen un éxito improbable o insuficiente a la luz de los conocimientos disponibles

4. Implementación cuidadosa de las alternativas para descubrir las partes críticas del método.

5. Seguimiento de la respuesta de los indicadores clave y análisis de los resultados en relación con los objetivos iniciales

6. Incorporación de los resultados a las decisiones futuras

Así lo que se pretende es que cada tratamiento (clareo, clara, poda, siembra,...) sea considerado como parte de un experimento a escala real que debe planificarse para obtener información útil para la gestión y así poder orientar actuaciones selvícolas futuras. Por lo tanto se deben superar los conceptos de parcelas permanentes, ensayos de procedencias o sitios experimentales para complementarlos, o en algunos casos sustituirlos, por 'experimentos' a escala real. Se trata, por tanto, de un sistema de aprendizaje mediante la práctica aprovechando los éxitos y fracasos tanto propios como ajenos.

Sin embargo, aunque este concepto de aprendizaje mediante la práctica es esencial en la aplicación de la gestión adaptativa, para que sea realmente útil no debe haber sólo una dimensión incremental del conocimiento. Debe haber un proceso formal, explícito y deliberado para aumentar el conocimiento útil de los gestores forestales a través de experimentos y el contraste, procesado crítico de los resultados y la aplicación de nuevas estrategias de gestión (StankeY et $a l .$, 2005). Por último, para que el aprendizaje sea real y para que tenga un claro impacto, debe tener dos dimensiones: una cognitiva (entendemos mejor los procesos que hemos estudiado) y otro conductual (cambiamos nuestra forma de hacer gestión forestal).

\section{Bases de la gestión adaptativa}

La base de la gestión adaptativa es que la gestión de comenzar a partir de experimentos a escala real con base en nuestro conocimiento científico. De este modo, las hipótesis subyacentes deben ser formuladas de forma rigurosa y posteriormente comprobadas (mediante el rechazo de la correspondiente hipótesis nula) y la validez de sus predicciones debe ser contrastada de forma que se mejore la gestión con los resultados obtenidos. La idea central es que la gestión de los sistemas naturales debe ser técnica pero con una base antropológica y ecológica. Por tanto la gestión forestal debe ser científicamente admisible, técnicamente posible, económica viable y socialmente aceptable (BrAVO, 1989).

\section{¿Cuáles son las principales razones para desarrollar un programa de gestión adaptativa?}

El principal motivo por el que se debe desarrollar un programa de gestión adaptativa es conseguir transformar la experiencia local obtenida, sin valor para determinar relaciones causales, en información útil para analizar los porqués de los resultados obtenidos. De esta forma podemos incrementar y consolidar el conocimiento técnico aplicado en la gestión forestal. Si consideramos que la situación de los bosques y el sector forestal en su conjunto no cambia, podríamos aplicar las técnicas habituales sin mayor problema (lo que se conoce en gestión pastoral como 'rutinas pastorales') y no haría falta una gran base científica o técnica, bastaría con una persona habilidosa para repetir lo que se hace habitualmente. Sin embargo, si las condiciones son cambiantes (como lo son en la actualidad) es necesario generar nuevo conocimiento para desarrollar nuevas alternativas selvícolas.

\section{Las oportunidades de la gestión adaptativa para el cambio de paradigma de la ciencia forestales}

La investigación selvícola debe centrarse en la indagación de los gradientes y los procesos subyacentes (PuetTMANN et $a l ., 2009$ ) para definir el ámbito de inferencia de los resultados, proveer información acerca de los beneficios relativos de cada tratamiento e incrementar las opciones de generalizar los resultados centrándose en los prin- 
cipios subyacentes. En muchos casos los trabajos de investigación se centran en problemas locales en lugar de, a partir de una situación local, intentar explicar un proceso de interés general. Esto limita el avance de la ciencia forestal e incluso, a la larga, que sea considerada como ciencia.

\section{Análisis de Hipótesis múltiples}

Mediante las bases de datos generadas con el desarrollo de programas de gestión adaptativa se puede disponer de información suficiente para realizar el análisis de hipótesis múltiples mediante la selección de modelos competitivos. FRANKLIN et al. (2001) describieron los siete aspectos claves de este tipo de análisis: 1) Desarrollar a priori de un conjunto de modelos candidatos cada uno de los cuales representa una hipótesis candidata, 2) Evaluar la estructura de los modelos y la consistencia de las variables incluidas, 3) Ajustar los modelos candidatos mediante máxima verosimilitud, 4) Establecer una jerarquía de los modelos a partir de índices de información (p.ej., Indice de divergencia de Kullback-Leibler, Criterio de Información de Akaike,...), 5) Examinar los modelos seleccionados en el paso anterior para comprobar la fortaleza de las inferencias en relación con las evidencias disponibles, 6) estimar la variación explicada por el o los modelos seleccionados y 7) Hacer inferencias formales a partir del o de los modelos seleccionados. El primer paso es clave pues sobre él se asienta la validez de este método. Bravo et al. (2001 y 2013) mostraron cómo mediante la inclusión de variables en modelos lineales y logísticos se pueden analizar varios modelos competitivos que representan hipótesis alternativas (en estos casos relacionados con el crecimiento, la mortalidad y la producción de piñas) que quieren ser comprobadas y, en su caso, seleccionadas. En este proceso el uso de estrategias de selección de variables paso a paso deben ser totalmente evitadas a favor de un proceso de inclusión de variables razonado y fundamentado

\section{Cuestiones que limitan de la gestión adaptativa para el cambio de paradigma de la ciencia forestales}

Las principales dificultades para implementar un programa de gestión forestal adaptativa depende en gran medida de las condiciones locales (ecológicas, administrativas, sociales, económicas,...) pero en general se pueden resumir en las siguientes:

1. Excesiva carga burocrática de las administraciones públicas.

2. Falta de medios económicos y técnicos en los bosques privados (esto es cierto los bosques mediterráneos pero puede no serlo en plantaciones forestales en otras áreas)

3. Falta de formación en técnicas estadísticas avanzadas (diseño de experimentos, técnicas bayesianas,...) y en métodos de tomas de decisión bajo incertidumbre.

4. Falta de conocimiento sobre la gestión adaptativa, sus métodos y técnicas

5. Falta sistemática de dudas en la gestión forestal

6. Falta de tradición en el intercambio de experiencia entre gestores y de éstos con los investigadores.

Aunque las limitaciones presupuestarias no debería constituir un problema insalvable, si los costos de la gestión adaptativa se integran en los planes de gestión, en algunos casos pueden servir de barrera para limitar su implementación justificando otras razones como dificultades administrativas o falta de disposición de los gestores

\section{¿POR QUÉ NO FUNCIONA LA TRANSFERENCIA TECNOLÓGICA EN EL SECTOR FORESTAL?}

Durante los últimos decenios se ha realizado un gran esfuerzo en investigación, desarrollo e innovación en el sector forestal. Sin embargo, no hay canales adecuados para que los investigadores comuniquen sus resultados de una forma efectiva o para que los gestores promuevan investigación que responda a sus necesidades. Por otro lado, el sistema científico promueve y premia la comunicación de resultados científicos entre investigadores (p.ej., mediante artículos científicos) pero no a los gestores. En muchos casos, las revistas científicas son de acceso difícil a los gestores porque solo están disponibles en bibliotecas especializadas o porque están escritas en inglés que es la lengua franca de la comunidad científica internacional. Además, los gestores encuentran barreras, económicas y de tiempo, para acudir a congresos científicos espe- 
cializados donde poder interactuar con los investigadores. Finalmente hay otros factores que deben ser considerados para explicar las dificultades en la transferencia tecnológica (FINCH \& PATTON-MALlORY, 1993):

1. Los resultados de investigación están normalmente dispersos y fragmentados en diversas publicaciones científicas y faltan publicaciones que sinteticen toda la información en forma de un conjunto de recomendaciones de gestión.

2. No existen procesos adecuados para identificar y priorizar los retos y preguntas científicas de forma que los resultados de la investigación pasen a formar parte del estado del arte.

3. Parte de la información científica tiene una utilidad limitada porque se centra en aspectos demasiado concretos.

4. Las rutinas y el ambiente de trabajo generan barreras filosóficas entre gestores e investigadores. Mientras que los investigadores trabajan, en general, con una gran libertad, los gestores tienen que convivir con la realidad política y económica en su día a día.

\section{CONCLUSIONES}

La puesta en marcha y desarrollo de un programa de gestión adaptativa precisa que los investigadores y los gestores trabajen juntos desde la planificación inicial de los tratamientos a partir de los cuales se pretende extraer el conocimiento científico (FINCH \& PATTON-MALLORY, 1993). Este punto es muy importante pues los gestores estarán más dispuestos a trasladar los resultados a los tratamientos selvícolas si sienten que han participado en su generación desde el principio. Todos los esfuerzos asociados a la gestión forestal adaptativa solo serán realmente útiles si los investigadores salen de sus círculos académicos y sus laboratorios para aproximarse a la gestión forestal. Por otro lado, los gestores deben cambiar su mentalidad y dejar de considerar la investigación como propia de círculos teóricos donde un grupo de científicos generan nuevos datos y conocimiento con limitada utilidad salvo dar un toque de modernidad a la gestión. La sociedad necesita que las decisiones técnicas se basen en conocimiento bien fundado y que sirvan para un entorno en continuo cambio. La ciencia y la gestión forestal no pueden seguir caminos paralelos y deben buscar puntos de encuentros que las refuercen mutuamente en beneficio de la sociedad en general y del sector forestal en particular. La investigación selvícola debería centrarse en las teorías generales e investigar la respuesta básica para desarrollar generalizaciones conceptuales basadas en la comprensión de los procesos subyacentes a las respuestas a los tratamientos (PUETTMANN et al., 2009) de este modo se pueden generar regímenes selvícolas flexibles y adaptados a las condiciones ambientales y sociales que confronta la gestión forestal en la actualidad.

\section{Agradecimientos}

El presente trabajo ha sido parcialmente financiado por el proyecto de investigación AGL2011-29701-C02-02 financiados por el Gobierno de España y FEDER-Fondo Europeo de Desarrollo Regional

\section{BIBLIOGRAFÍA}

Bravo, F.; 1989. Estudio silvopastoral de la dehesa boyal de Alía (Cáceres). Ecología 3: 107-115

Bravo, F.; (Coord) 2007. El papel de los bosques españoles en la mitigación del cambio climático. Fundación Gas Natural. Madrid.

Bravo, F.; HanN, D. \& Maguire, D.A.; 2001. Impact competitor species composition on predicting diameter growth and survival rates of Douglas-fir trees in southwest Oregon. Can. J. For. Res. 31: 2237-2247

Bravo, F.; GonzÁlez-Martínez, S.C. \& MaguIRE, D.; 2013. Relación entre el crecimiento radial y la producción de piñas bajo diferentes condiciones climáticas: El caso de Pinus pinaster Ait. en la Meseta Castellana. En: Actas del $6^{\circ}$ Congreso Forestal Español. S.E.C.F. Disponible en: http://www.congresoforestal.es/fichero.php? $\mathrm{t}=41725 \& \mathrm{i}=4887 \& \mathrm{~m}=2185$

National Research COUNCIL; 2002. National Capacity in Forestry Research. The National 
Academies Press. Washington, DC. Disponible en http://www.nap.edu/openbook.php?record_id=10384

Helms, J.A.; 1998. Dictionary of Forestry. Society of American Forestry. Disponible en: http://www.dictionaryofforestry.org/

Puettmann, K.J.; Coates, K.D. \& Messier, C.; 2009. A critique of silviculture. Managing for complexity. Island Press. Washington.

LEARY, R.A.; 1985. Interaction theory in forest ecology and management. Kluwer Pub. Dordrecht.

Deming, W.E.; 1993. The New Economics for Industry, Government, Education. Cambridge: Massachusetts Institute of Technology Center for Advanced Engineering Study. Cambridge.

Finch, D.M. \& Patton-Mallory, M.; 1993. Closing the gap between research and management In: D.M. Finch \& P.W. Stangel (eds.). Status and management of neotropical migratory birds: 12-16. Estes Park, Colorado. Gen. Tech. Rep. RM-229. Rocky
Mountain Forest and Range Experiment Station, U.S. Dept. of Agriculture, Forest Service. Fort Collins.

NYBERG, J.B.; 1998. Statistics and the practice of adaptive management. In: V. Sit, V. \& B. Taylor (eds), Statistical methods for adaptive management studies. Land Manage. Handb. 42: 1-7. Res. Br., B.C. Min. For., Res. Br.

StankeY. G.H.; Clark, R.N. \& Bormann, B.T.; 2005. Adaptive management of natural resources: Theory, concepts and management institutions. Gen. Tech. Rep PNPWGTR-654. U.S. Department of Agriculture, Forest Service, Pacific Northwest Research Station. Portland.

Holling, C.S.; 1978. Adaptive environmental assessment and management. John Wiley. London.

Real ACAdEMia DE Ingeniería; 2014. Diccionario Español de Ingeniería. http://diccionario.raing.es Versión 1.0. [último acceso 3 de septiembre de 2014]. 